\title{
Effect of fluid flow on solidified equiaxed dendrite morphology evolution based on phase field - lattice Boltzmann method
}

\author{
Wei-zhao Sun', Rui Yan', Xiong Wan', Hong-biao Dong', *Tao Jing' \\ 1. Key Laboratory for Advanced Materials Processing Technology, Ministry of Education, School of Materials Science and Engineering, \\ Tsinghua University, Beijing 100084, China \\ 2. Department of Engineering, University of Leicester, LE1 7RH, UK
}

\begin{abstract}
Fluid flow can significantly change the evolution of microstructural morphology. However, relatively little is known how the fluid flow, concentration and microstructure affect each other quantitatively, which is essential to optimize processing parameters. A quantitative simulation study of Al-Cu solidified equiaxed dendrite evolution under forced flow based on phase field - lattice Boltzmann method (PF-LBM) is carried out. Results obtained are validated by Gibbs-Thomson relation at the dendrite tip. Compared with the equiaxed dendrite evolution without flow, the upstream dendrite arm is enhanced while the downstream arm is inhibited. Besides, as the inlet flow rate increases, the secondary arms attached onto the upstream primary arm and the upstream side of the primary arm normal to the inflow velocity has been well developed. Results show that sidewise instabilities of the primary dendrite arm and onset of secondary arm is caused by the local concentration perturbation and will be enhanced or inhabited by the flow. It is believed that the coupled PF-LBM method is able to handle dendrite evolution under forced flow quantitatively, which helps in investigating the solidified dendrite morphology evolution.
\end{abstract}

Key words: phase field; lattice Boltzmann method; forced flow; instability

CLC numbers: TP391.9 Document code: A Article ID: 1672-6421(2018 06-422-06

Nolidification is one of the most universal phenomena that occurs in numerous processing procedures, such as casting, welding and additive manufacturing ${ }^{[1]}$. It is widely accepted that the solidified microstructure, which is predominated by dendrites, mainly determines the performance of final components ${ }^{[2]}$. Predicting microstructure evolution by simulations with processing parameters involved, especially those in which complicated heat and solute diffusion and fluid flow are coupled, has become a promising method for optimization of processing parameters to obtain a desired microstructure.

Earlier studies were mainly focused on heat or solute diffusional phase transformations ${ }^{[3-5]}$. However, convection caused by natural or forced flow, which is inevitable in real situations and frequently plays a significant role in microstructure evolution, has been

\section{* Tao Jing}

Male, born in 1965, Professor. His research interests mainly focus on materials processing technology and integrated computational material engineering (ICME). He has published more than 100 papers.

E-mail: jingtao@mail.tsinghua.edu.cn

Received: 2018-06-22; Accepted: 2018-09-05 ignored due to its complexity. Some experimental studies ${ }^{[6,7]}$ have been conducted to investigate how the flow influences the morphology evolution. Badillo et al. ${ }^{[6]}$ conducted experiments about the equiaxed crystals of the transparent model alloy succinonitrileacetone which developed in an undercooled melt. It was found that the upstream dendrite tip growth velocity increases, while the average of six dendrites' tip growth velocity keeps constant under forced flow. Shevchenko et al. ${ }^{[7]}$ investigated the directional solidification of Ga$25 \mathrm{wt} . \%$ In alloy under natural flow by means of X-ray radioscopy. Lighter solute $\mathrm{In}$ was rejected from the solid into the liquid, and the buoyance-driven convection, i.e. natural convection, was produced, which in turn significantly affected the microstructure. However, much less numerical research ${ }^{[8-11]}$ has been done due to limited development of the computational method with both the microstructure evolution and the fluid flow dynamics involved. Sun et al. ${ }^{[8]}$ developed a twodimensional (2D) cellular automation (CA) - lattice Boltzmann method (LBM) to simulate the dendritic growth of binary alloys under forced flow. It was demonstrated that the dendritic growth was strongly 
influenced by convection. Similar studies have also been done by Takaki et al ${ }^{[9,10]}$, wherein phase field (PF) method ${ }^{[12]}$ was employed to simulate the microstructure evolution. Qi et al. ${ }^{[1]}$ developed a PF-Naiver-Stockers (NS) method to investigate the motion of equiaxed dendritic crystals under natural and forced flow.

In order to model dendrite evolution under flow, the mathematical model should be composed of two aspects. One is for describing the solid-liquid (S-L) phase transformation, and the other is for fluid flow. For S-L phase transformations, the PF method has become the most popular method to simulate microstructure evolution, owing that explicit interface tracking is avoided, which shows exceptional advantages especially for three-dimensional dendrite evolution with complicated topological relations. In the PF method, a new parameter called order parameter $\Phi$ is introduced to specify different phases, as in the liquid phase $\Phi=-1$ and the solid phase $\Phi=1$. At the S-L interface, $\Phi$ is interpolated by the interpolation function, which is constructed delicately, and varied smoothly but sharply. The PF model can be reduced as Gibbs-Thomson relation by asymptotic matched analysis ${ }^{[13]}$. As for the fluid flow calculation, there are mainly two available methods - the NS solver ${ }^{[11]}$ and the $\mathrm{LBM}^{[9]}$. At mesoscopic scale, the LBM shows advantages over the NS equations. It has been shown that the fluid flow calculations using a NS solver no longer converged when the solid volume fraction went above $0.3^{[8]}$. However, instead of simulating the transport equations (heat, mass and momentum) in a continuum way (the NS equations) or considering every particle (molecular dynamics), a distribution function which is used to describe characters of a collection of particles is utilized in the LBM. The transport of distribution function can be considered as collision and then streaming. The LBM is also a robust method and can be transformed into NS solver when the parameters are chosen properly.

In this work, PF-LBM was employed to quantitatively simulate the dendrite evolution under forced flow. Taking the $\mathrm{Al}-\mathrm{Cu}$ alloy as an example, the model is quantitatively validated by Gibbs-Thomson equation at the dendrite tip. The dendrite growth and stability are also discussed.

\section{Method}

\subsection{Phase field model}

The quantitative PF model was proposed by Echebarria et $\mathrm{a}^{[5]}$, which was originally intended to be employed in simulations of directional solidification. In order to simulate equiaxed dendrite evolution, the driving force term was rewritten, in which the directional undercooling was substituted by a unified undercooling.

The 2D governing equations for order parameter $\Phi$ and solute $c$ with the undercooling $\Delta T_{0}(>0)$ are:

$$
\begin{aligned}
\tau(\vec{n}) \frac{d \phi}{d t}=\boldsymbol{\nabla} & \cdot\left(W(\vec{n})^{2}\right) \nabla \phi+\frac{\partial}{\partial x}\left(|\nabla \phi|^{2} W(\vec{n}) \frac{\partial W(\vec{n})}{\partial \phi_{x}}\right)+\frac{\partial}{\partial y}\left(|\nabla \phi|^{2} W(\vec{n}) \frac{\partial W(\vec{n})}{\partial \phi_{y}}\right) \\
& -f^{\prime}(\phi)+\lambda g^{\prime}(\phi)\left(\frac{\Delta T_{0}}{m_{l} c_{l}^{0}(k-1)}-\frac{e^{u}-1}{1-k}\right)
\end{aligned}
$$

$$
\frac{d c}{d t}=\boldsymbol{\nabla} \cdot(D q(\phi) c \boldsymbol{\nabla} u)+\boldsymbol{\nabla} \cdot \boldsymbol{j}_{A T}+\boldsymbol{\nabla} \boldsymbol{j}_{N}
$$

where $\vec{n}$ is the unit crystal orientation; $\Phi_{x(y)}$ is the derivative of $\Phi$ with respect to $x$ or $y ; m_{1}, c_{1}^{0}$ and $k$ are the liquidus slope, initial liquid solute concentration and partition coefficient, respectively; $D$ is the diffusion coefficient in the liquid; $\boldsymbol{\nabla}=\left(\frac{\partial}{\partial x}, \frac{\partial}{\partial y}\right)$ is the 2D nabla operator. $\tau(\vec{n})=\tau_{0} a^{2}(\vec{n})$ is the relaxation time, where $a(\vec{n})=1+\varepsilon_{4}\left[\frac{\phi_{x}^{4}+\phi_{y}^{4}}{\left(\phi_{x}^{2}+\phi_{y}^{2}\right)^{2}}-3\right]$ is introduced to represent the crystalline anisotropy, $\varepsilon_{4}$ is the anisotropic strength, $\tau_{0}=a_{2}\left(\lambda W_{0}^{2}\right) / D$ is the unit of relaxation time and $a_{2}=0.6267$ is constant. $W(\vec{n})=W_{0} a(\vec{n})$ is the interface width. $f(\Phi)=-\frac{1}{2} \Phi^{2}+\frac{1}{3} \Phi^{3}$ and $g(\Phi)=\Phi-\frac{2}{3} \Phi^{3}+\frac{1}{5} \Phi^{5}$ are the interpolation functions associated with the double-well potential and the bulk free energy, respectively, and $f^{\prime}(\Phi), g^{\prime}(\Phi)$ are the derivatives. $q(\Phi)=(1-\Phi) / 2$ is also the interpolation function to interpolate the diffusion coefficient from the solid to liquid. $\lambda=a_{1} W_{0} / d$ is a coupling parameter to rescale the energy caused by the solute, where $a_{1}=5 \sqrt{2} / 8$ is a constant and $\mathrm{d}$ is the chemical capillary length. $\boldsymbol{j}_{\boldsymbol{A} T}$ and $\boldsymbol{j}_{N}$ are the anti-solute trapping ${ }^{[14]}$ and noise perturbation terms ${ }^{[15]}$, respectively. The dimensionless concentration $u_{i}$ is the departure of concentration from equilibrium. Further details can be found in Ref. ${ }^{[12]}$.

\subsection{Lattice Boltzmann method}

The motion and rotation of dendrites were ignored. Therefore, $d \Phi / d t$ in the left hand of Eq. (1) reduces to $\partial \Phi / \partial t$. However, the solute in the liquid is influenced by the fluid flow, and thus $d c / d t$ in the left hand of Eq. (2) expands into $\partial c / \partial t+\boldsymbol{U} \cdot \nabla c$, where $\boldsymbol{U}$ is the local velocity of the fluid. For the solid, $\boldsymbol{U}=0$ is obtained and $d c / d t$ reduces to $\partial c / \partial t$, which is consistent with the real physics process. When the liquid runs into the solid, bounce boundary is employed. To avoid explicit tracking the S-L interface, Beckermann et $\mathrm{al}^{[16]}$ added an additional discrete force $G_{i}(x, t)$ in the fluid flow equations. $G_{i}(x, t)$ is obtained by analogizing to the fluid flow past porous materials and the reaction force is put back into the liquid. Therefore, the LBM equation ${ }^{[9]}$ with BGK (Bhatnagar-Gross-Krook) approximation and $G_{i}(x, t)$ is

$$
f_{i}\left(\boldsymbol{x}+\boldsymbol{c}_{i} \delta t, t+\delta t\right)=f_{i}(\boldsymbol{x}, t)-\frac{\delta t}{\tau_{l}}\left[f_{i}(\boldsymbol{x}, t)-f_{i}^{e q}(\boldsymbol{x}, t)\right]+G_{i}(\boldsymbol{x}, t) \delta t
$$

where $f_{i}(x, t)$ is the distribution function in the $i$ th direction from a statistical physics point of view, $f_{i}^{\text {eq }}(x, t)$ is the equilibrium distribution function, $\boldsymbol{c}_{i}$ is the velocity in the $i$ th direction, and $t$, $\delta t$ and $\tau_{l}$ are the time, time interval and relaxation time for LBM, respectively. It should be pointed out that the relaxation time $\tau_{l}$ in LBM is not the same as that in PF and is related to the fluid viscosity $v$,

$$
v=\frac{1}{3}\left(\frac{\delta x}{\delta t}\right)^{2}\left(\tau_{l}-\frac{\delta t}{2}\right)
$$

The fluid density $\rho$ and flow velocity $\boldsymbol{U}$ are able to obtained by

$$
\rho=\sum_{i=0}^{Q-1} f_{i}
$$




$$
\rho \boldsymbol{U}=\sum_{i=0}^{Q-1} c_{i} f_{i}
$$

where $Q$ is the number of discrete velocity. In this study, a 2D-nine-veloctiy (D2Q9) lattice arrangement is employed. The nine discrete velocities $c_{i}$ are given by

$c_{i}=\left\{\begin{array}{cc}(0,0), \quad \text { for } i=0 & \\ c\left(\cos \left[\frac{(i-1) \pi}{2}\right], \sin \left[\frac{(i-1) \pi}{2}\right]\right), & \text { for } i=1-4 \\ \sqrt{2} c\left(\cos \left[\frac{(2 i-9) \pi}{4}\right], \sin \left[\frac{(2 i-9) \pi}{4}\right]\right), & \text { for } i=5-8\end{array}\right.$

where $c=\delta x / \delta t$ is the lattice velocity. The equilibrium function is given by

$$
f_{i}^{e q}(\boldsymbol{x}, t)=w_{i} \rho\left[1+\frac{3\left(\boldsymbol{c}_{i} \cdot \boldsymbol{U}\right)}{c^{2}}+\frac{9\left(\boldsymbol{c}_{\boldsymbol{i}} \cdot \boldsymbol{U}\right)^{2}}{2 c^{4}}-\frac{3 \boldsymbol{U} \cdot \boldsymbol{U}}{2 c^{2}}\right]
$$

where $w_{i}$ is the weight coefficient, which are $4 / 9$ for $i=0,1 / 9$ for $i=1-4$ and $1 / 36$ for $i=5-8$, respectively. When additional discrete force $G_{i}(x, t) \delta t$ is applied, an increment $\boldsymbol{\Delta} \boldsymbol{U}$ of velocity is generated, and thus the equilibrium is perturbed ${ }^{[17]}$. Therefore, with higher order infinitesimals neglected, the last term in Eq. (3) is

$$
\begin{aligned}
G_{i}(\boldsymbol{x}, t) \delta t & =f_{k}^{e q}(\rho, \boldsymbol{U}+\Delta \boldsymbol{U})-f_{k}^{e q}(\rho, \boldsymbol{U}) \\
& =w_{i} \rho\left[3 \frac{\boldsymbol{c}_{\boldsymbol{i}}-\boldsymbol{U}}{c^{2}}+9 \frac{\left(\boldsymbol{c}_{\boldsymbol{i}} \cdot \boldsymbol{U}\right) \boldsymbol{c}_{\boldsymbol{i}}}{c^{4}}\right] \cdot \Delta \boldsymbol{U}
\end{aligned}
$$

According to Ref. ${ }^{[16]}, \boldsymbol{U} \boldsymbol{U}$ is given by

$$
\Delta \boldsymbol{U}=-\frac{2 v g}{W_{0}^{2}}\left(\frac{1+\phi}{2}\right)^{2} \boldsymbol{U} \delta t
$$

To avoid complicated programming, $\delta x$ and $\delta t$ in the LBM were the same as the unified grid spacing $d x$ and time interval $d t$ in the PF method, respectively. In other words, the grid for the LBM was anchored to that for the PF method.

\section{Results and discussions}

The alloy employed in this study is $\mathrm{Al}-4 \mathrm{wt} . \% \mathrm{Cu}$. Therefore, the initial solute concentration in the liquid $c_{l}^{0}$ was 0.04 . Physical parameters used are listed in Table $1^{[11]}$. The anisotropic strength used here was 0.03 , while the real one is around 0.01 . It should be pointed out that our main concern is not to simulate the dendrite precisely but to discover how the fluid flow influences the microstructure evolution. The artificial magnified anisotropic strength enhances the side branching, accelerates the simulation speed and does not influence the underlying mechanisms within limited computational domain.

The PF-LBM was solved in a dimensionless form, in which the spatial length was in unit of interface width $W_{0}$ and the temporal length was in unit of relaxation time $\tau_{0}$ for the PF method. The ratio of $W_{0}$ to chemical capillary length $d=\Gamma /\left(m_{l}(k-1) c_{l}^{0}\right)=$ $2.68 \times 10^{-2} \mu \mathrm{m}$ was set as 5, i.e., $W_{0}=5 d=1.342 \times 10^{-1} \mu \mathrm{m}$. The adiabatic boundary was applied longitudinally while periodic boundary was applied vertically for the PF model. For the
Table 1: Physical parameters of Al-Cu alloy ${ }^{[11]}$

\begin{tabular}{lc}
\multicolumn{1}{c}{ Parameters } & Value \\
\hline Liquidus slope, $m_{l}\left[\mathrm{~K} \cdot(\mathrm{wt} . \%)^{-1}\right]$ & -260 \\
Liquidus diffusion coefficient, $D\left[\mathrm{~m}^{2} \cdot \mathrm{s}^{-1}\right]$ & $3.0 \times 10^{-9}$ \\
Partition coefficient, $k$ & 0.14 \\
Gibbs-Thomson coefficient, $\Gamma[\mathrm{m} \cdot \mathrm{K}]$ & $2.4 \times 10^{-7}$ \\
Anisotropic strength, $\varepsilon_{4}$ & 0.03 \\
Fluid viscosity, $v\left[\mathrm{~m}^{2} \cdot \mathrm{s}^{-1}\right]$ & $5 \times 10^{-7}$
\end{tabular}

LBM, to generate forced flow, a unified constant flow rate was introduced on the left boundary, which could be regarded as the inlet. Free boundary condition was adopted on the right side, and bounce boundary condition on both the top and bottom sides for the LBM. Letting $d x=W_{0}$ and $d t=\left(d x^{2}\right) / 5 D=4.8 \times 10^{-2} \mu$ s, the scales used for length and time are in unit of $\mathrm{dx}$ and $\mathrm{dt}$, respectively, without further statements. All cases were carried out with the simulation size of $1,024 \times 1,024$ and simulation time of 30,000 steps. A nucleation seed was put at the center of the simulation domain with a constant undercooling $\Delta T_{0}=5 \mathrm{~K}$. In order to investigate the influence of forced flow, five inlet flow rates were set to be 0.00 (without forced flow), 0.05, 0.10, 0.15, and 0.20 in the unit of $d x / d t$, respectively.

\subsection{Validation}

The results of the simulation were validated by Gibbs-Thomson equation at the dendrite tip. To quantitatively evaluate the derivation of the calculated results from analytical results, a new dimensionless variable $\Delta_{G T \text { error }}$ is defined ${ }^{[18]}$, i.e.

$$
\Delta_{\text {GTerror }}=\frac{\left(1-15 \varepsilon_{4}\right) \Gamma \kappa-m_{l}\left(c_{l}^{*}-c_{l}^{0}\right)}{\Delta T_{0}}-1
$$

where $\kappa$ is the curvature and $c_{l}{ }^{*}$ is the solute concentration on the liquid side at the dendrite tip. $c_{l}{ }^{*}$ is deduced from $c_{s}{ }^{*} / k$, where $c_{s}^{*}$ is the solute concentration in the solid side. Using this approach, the concentration in the liquid is much more precise. The dendrite tip curvature is fitted by the contour of $\phi=0$. There is no map relation for S-L interface between the sharp-interface model and PF model at the $\Phi=0$ contour. Therefore, some fluctuations might occur and the simulation is reliable if the deviations are smaller than the upper limit.

The calculated results were extracted at the last time step for all the cases. The variation of $\Delta_{\text {GTerror }}$ is shown in Fig. 1. The derivation varies around $-3 \%$, demonstrating that high accuracy of the simulation was obtained. It is unambiguous to announce that the PF results are correct within limited deviation.

\subsection{Quantitative analysis for influence of fluid}

The morphology evolution of equiaxed dendrite for several selected time steps is given in Fig. 2, with the upper panel showing the case of inlet flow rate equal to 0.20 and the lower panel showing the case of inlet flow rate equal to 0.00 . To show the difference clearly, only half of the symmetrical dendrite is presented. It can be seen clearly that the flow significantly 


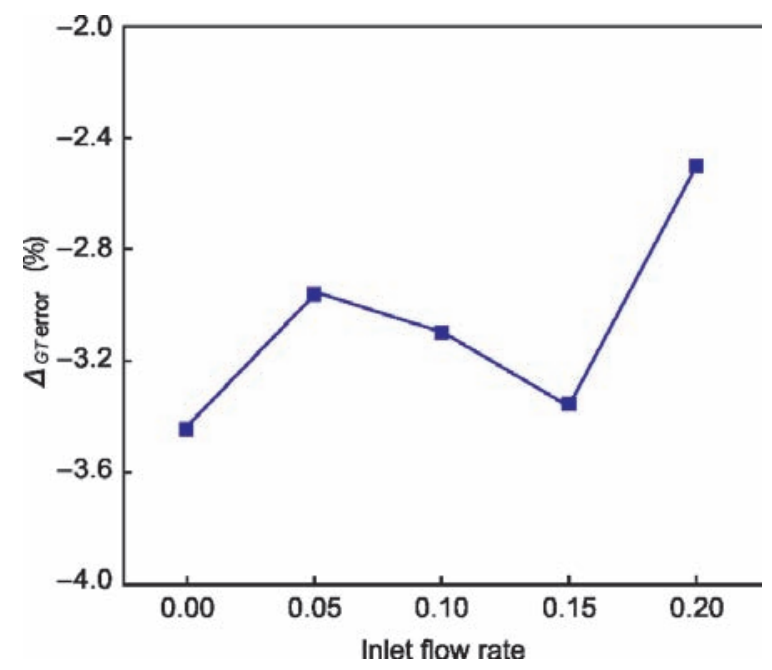

Fig. 1: Variation of $\Delta_{\text {GTerror }}$ as a function of inlet flow rate

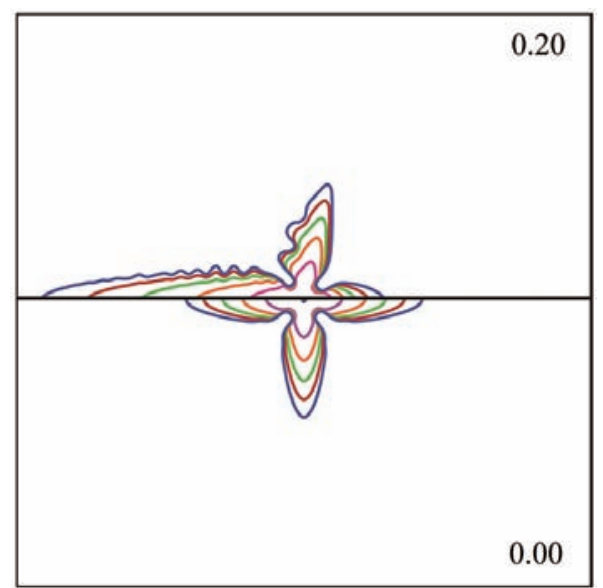

Fig. 2: Dendrite evolution at time step of $6 \times 10^{3}$ (purple line), $1.2 \times 10^{4}$ (orange line), $1.8 \times 10^{4}$ (green line), $2.4 \times 10^{4}$ (red line) and $3.0 \times 10^{4}$ (blue line) for inlet flow rate 0.20 (upper panel) and 0.00 (lower panel)

influences the morphology evolution, leaving the upstream dendrite arm enhanced and the downstream arm inhibited. The arms normal to the inlet flow tilt, while their lengths remain about the same as that in the lower panel. The qualitative analytical results are consistent with experiments observations ${ }^{[6]}$.

In order to quantitatively describe the influence of the flow, the tip growth rates of dendrite arms were compared in two cases, one with the inlet flow rate equal to 0.00 and the other with an inlet flow rate equal to 0.02 . The results are shown in Fig. 3. In the case where force flow is absent, due to fourfold symmetry of the dendrite, the tip growth rate of one dendrite arm was extracted (the orange line in Fig. 3), reaching around $v_{N}=0.56 \times 10^{-2}$ at stage where stable growth has achieved. For the case in which the inlet flow rate is equal to 0.02 , four different dendrite arm growth rates were obtained. For the left arm (the red line in Fig. 3), i.e. the upstream arm, stable growth rate increases to $v_{L}=1.60 \times 10^{-2}$, while for the right arm (the green line in Fig. 3), i.e. the downstream arm, stable growth rate could not be obtained and decreases continuously, the reason for which will be discussed later. For the top $\left(v_{T}=0.59 \times 10^{-2}\right.$, the blue line in Fig. 3$)$ and bottom arm $\left(v_{B}=0.60 \times 10^{-2}\right.$, the purple line in Fig.

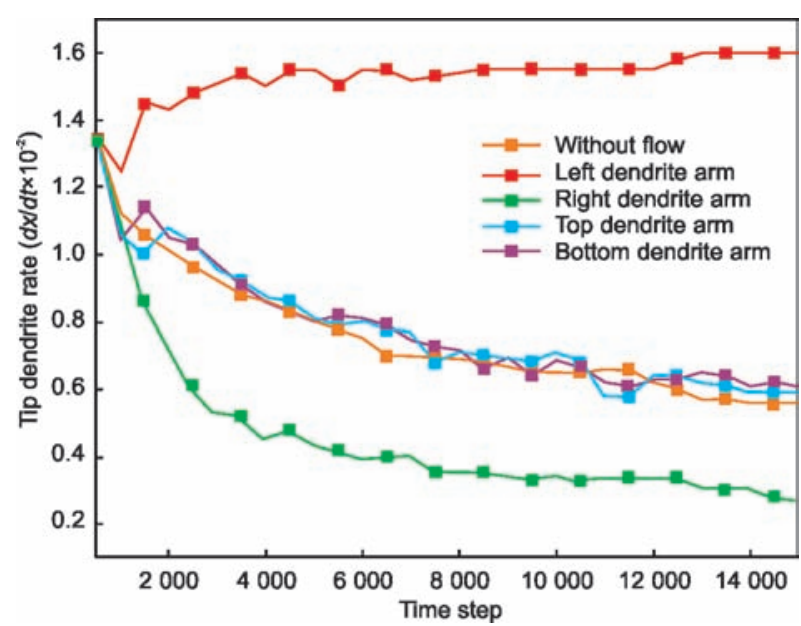

Fig. 3: Dendrite tip growth rates. The growth rate in the case without flow is presented by orange line. The left, right, top and bottom dendrite growth rates under forced flow with flow rate equal to 0.02 are denoted by red, green, blue and purple lines, separately

3) those are normal to the inlet flow velocity, stable growth rates are nearly identical with $v_{N}$.

Besides the growth rate of the dendrite tip which only reflects one-dimensional evolution, curvature radiuses and concentrations at primary dendrites were also analyzed to reveal 2D evolution of dendrites. As can be seen from Fig. 2, the evolution tendency of the tip radius is similar with that of the growth rate. For the sake of brevity, the evolution for tip radius as a function of time step is not displayed here. Instead, the quantitative information about the radii and concentrations at a particular time step of 30,000 was compared. The radius and dimensionless concentration at the tip of primary dendrite in the case without flow are 5.13 and 1.456 , respectively. In the case with inlet flow rate equal to 0.02 , the curvature radiuses are 3.203 (left), 6.019 (right), 5.295 (top) and 5.085 (bottom), and dimensionless concentrations are 1.446 (left), 1.465 (right), 1.454 (top) and 1.454 (bottom). Therefore, it can be concluded that the upstream tip curvature becomes sharper while the downstream becomes blunter. This means that the upstream dendrite is less affected by the concentrated solute transported from the neighbor solid phase as a result of the asymmetrical concentration distribution, which will be discussed later.

The difference in dendrite morphologies can be explained in terms of constitutional undercooling which is resulted from fluid flow. In the case without flow [Fig. 4(a)], both the dendrite morphology and the concentration distribution show fourfoldsymmetry. In the case with the inlet flow rate of 0.02 [Fig. 4(b)], in the direction of upstream, the rejected solute is transported away by the fluid. Therefore, the departure of concentration $\mathrm{u}$ in Eq. (1) at the dendrite tip decreases, i.e., the driving force increases. As a result, the growth of the upstream arm accelerates. In the direction of downstream, vortexes form and the rejected solute is mainly trapped in areas where the vortexes exist. As the rejected solute accumulates, the downstream arm growth rate decreases continuously. The top and bottom arms are normal to the inlet flow, so the concentration distribution at 
the dendrite tips does not change severely, leaving the growth rates of the top and bottom arms almost unchanged compared with $v_{N}$.

\subsection{Instability of secondary arm}

It can be seen from Fig. 4(b) that the secondary arms also appeared. The secondary arms attached to the upstream primary arm and the upstream side of the primary arm normal to the inflow velocity are well developed. Similar phenomena were also observed in cases with other inlet flow rates (Fig. 5), showing that secondary arms become more and more obvious as the inlet flow rate increases. According to the classical MullinSekerka theory ${ }^{[19]}$, the instability of the dendrite arms and the onset of secondary arms are caused by the local concentration perturbation and further enhanced by the force flow. Two aspects should be included, i.e., (a) the onset and (b) the development of secondary arms. (a) When local perturbations occur, secondary arm "buds" form. The perturbations are represented by the noise term $j_{N}$ in Eq. (2). (b) However, when the "buds" grow into areas with high concentration of rejected solute, the local driving force decreases, and therefore the growth rate slows down. In this situation, it is possible that the "buds" advance at the same speed as their solidified surroundings and the stability of the dendrite arm is still preserved. When local fluid flow exists, the local solute is diluted or concentrated depending on the inflow and outflow concentration of solute. For the "buds" under the red arrow 1 in Fig. 4(b), the dilute inflow decreases the concentration of the rejected solute, i.e., local driving force increases, and therefore, the "buds" are more likely to be well developed. For the "buds" under the red arrow 2, although the inflow is concentrated, the concentrated solution is then transported away by the fluid to downstream. In other words, the net increase is positive, i.e., local driving force still increases. For the "buds" under the red arrow 3 and arrow 4, due to the formation of vortexes, the inflow and outflow are both concentrated solution and the concentrated solute is restricted in the vortexes. Therefore, the "buds" are unlikely to develop. As illustrated in Fig. 5, the above deduction is also consistent with the instability trend as the inflow rate increases, i.e., as the inflow rate increases, the secondary arms developed mature.
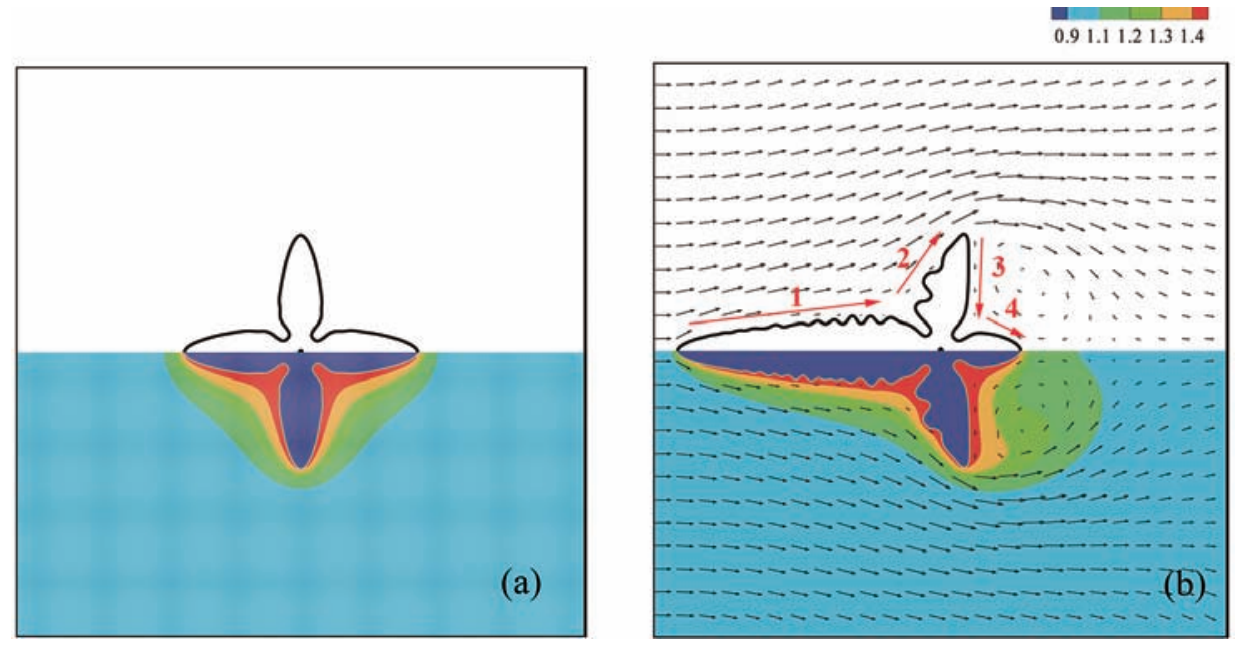

Fig. 4: Dendrite morphology (top panel) and concentration distribution (bottom panel) at time step of $3 \times 10^{4}$ for the case without flow (a) and the case with inlet flow rate 0.02 (b), the velocity vectors of the liquid are also presented. The red arrows in (b) denote the general direction of flow.

\section{Conclusions}

To investigate the influence of forced flow on the dendrite evolution, a study based on phase field - lattice Boltzmann method was employed to simulate equiaxed dendrite evolution. Five cases with different inlet flow rates $(0.00,0.05,0.10,0.15$ and 0.20$)$ were considered. The model was validated by the Gibbs-Thomson relation at the dendrite tip, showing that high accuracy was obtained. Consistent with the previous experiment results, we found that the upstream primary dendrite arm is enhanced while the downstream arm is inhibited by the forced flow. Based on quantitative analysis, it is found that the solute rejected from the solid can be transported away by the fluid flow. The underlying cause for the instability of primary dendrite arms and onset of secondary arms is also discussed. The solution can be diluted or concentrated by the flow, which affects the driving

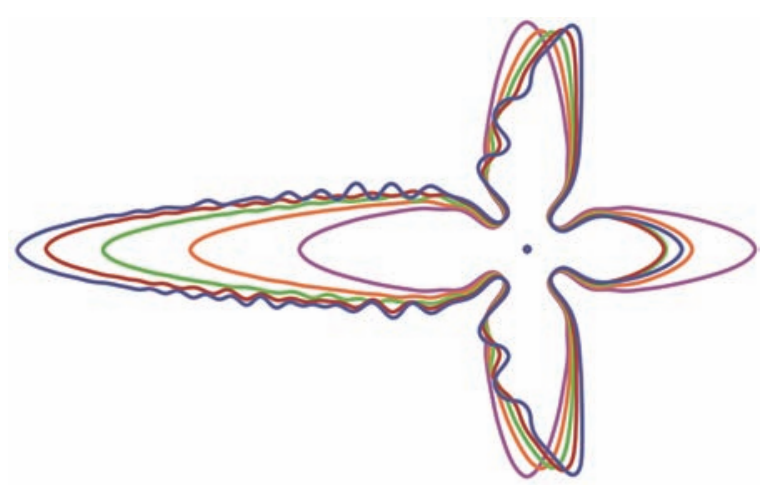

Fig. 5: Eventual dendrite morphologies in cases with inlet flow rate of $\mathbf{0 . 0 0}$ (purple line), $\mathbf{0 . 0 5}$ (orange line), 0.10 (green line), 0.15 (red line) and 0.20 (blue line), respectively 
force of solidification and eventually leads to the difference in the development of secondary arms. The coupled PF-LBM method provides a quantitative approach to handle dendrite evolution under forced flow, which will promote studies of dendrite morphology evolution under wider conditions.

\section{References}

[1] DebRoy T, Wei H, Zuback J, et al. Additive manufacturing of metallic components-process, structure and properties. Progress in Materials Science, 2017,

[2] Asta M, Beckermann C, Karma A, et al. Solidification microstructures and solid-state parallels: Recent developments, future directions. Acta Materialia, 2009, 57: 941-971.

[3] Jackson K, Hunt J. Lamellar and rod eutectic growth. Dynamics of Curved Fronts. Elsevier, 1988: 363-376.

[4] Kurz W, Giovanola B, Trivedi R. Theory of microstructural development during rapid solidification. Acta metallurgica, 1986, 34: 823-830.

[5] Dong H, Lee PD. Simulation of the columnar-to-equiaxed transition in directionally solidified Al-Cu alloys, Acta Materialia, 2005, 53: 659-668.

[6] Badillo A, Ceynar D, Beckermann C. Growth of equiaxed dendritic crystals settling in an undercooled melt, Part 1: Tip kinetics. Journal of Crystal Growth, 2007, 309: 197-215.

[7] Shevchenko N, Roshchupkina O, Sokolova O, et al. The effect of natural and forced melt convection on dendritic solidification in Ga-In alloys. Journal of Crystal Growth, 2015, 417: 1-8.

[8] Sun D, Zhu M, Pan S, et al. Lattice Boltzmann modeling of dendritic growth in a forced melt convection. Acta Materialia, 2009, 57: 1755-1767.
[9] Rojas R, Takaki T, Ohno M. A phase-field-lattice Boltzmann method for modeling motion and growth of a dendrite for binary alloy solidification in the presence of melt convection. Journal of Computational Physics, 2015, 298: 29-40.

[10] Takaki T, Sato R, Rojas R, et al. Phase-field lattice Boltzmann simulations of multiple dendrite growth with motion, collision, and coalescence and subsequent grain growth. Computational Materials Science, 2018, 147: 124-131.

[11] Qi X B, Chen $Y$, Kang X H, et al. Modeling of coupled motion and growth interaction of equiaxed dendritic crystals in a binary alloy during solidification. Scientific Reports, 2017, 7: 45770.

[12] Echebarria B, Folch R, Karma A, et al. Quantitative phasefield model of alloy solidification. Physical Review E, 2004, 70: 061604.

[13] Karma A, Rappel W-J. Quantitative phase-field modeling of dendritic growth in two and three dimensions. Physical Review E, 1998, 57: 4323.

[14] Karma A. Phase-field formulation for quantitative modeling of alloy solidification. Physical Review Letters, 2001, 87: 115701.

[15] Echebarria B, Karma A, Gurevich S. Onset of sidebranching in directional solidification. Physical Review E, 2010, 81: 021608.

[16] Beckermann C, Diepers H-J, Steinbach I, et al. Modeling melt convection in phase-field simulations of solidification. Journal of Computational Physics, 1999, 154: 468-496.

[17] Medvedev D, Fischaleck T, Kassner K. Influence of external flows on crystal growth: Numerical investigation. Physical Review E, 2006, 74: 031606.

[18] Ohno M. Quantitative phase-field modeling of nonisothermal solidification in dilute multicomponent alloys with arbitrary diffusivities. Physical Review E, 2012, 86: 051603.

[19] Mullins WW, Sekerka R. Stability of a planar interface during solidification of a dilute binary alloy. Dynamics of Curved Fronts. Elsevier, 1988: 345-352.

This research was financially supported by the National Key Research and Development Program of China (Grant No. 2017YFB1103700), and the National Science Foundation of China (Grant Nos. 51320105003 and 51674153). 